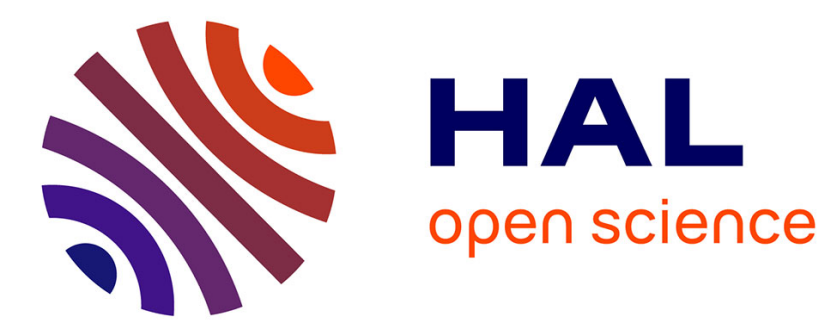

\title{
Global position-feedback tracking control of flexible-joint robots
}

\author{
Sofia Avila-Becerril, Antonio Loria, Elena Panteley
}

\section{To cite this version:}

Sofia Avila-Becerril, Antonio Loria, Elena Panteley. Global position-feedback tracking control of flexible-joint robots. 2016 American Control Conference (ACC), Jul 2016, Boston, MA, United States. pp.3008-3013, 10.1109/ACC.2016.7525377 . hal-01357298

\section{HAL Id: hal-01357298 \\ https://hal.science/hal-01357298}

Submitted on 29 Aug 2016

HAL is a multi-disciplinary open access archive for the deposit and dissemination of scientific research documents, whether they are published or not. The documents may come from teaching and research institutions in France or abroad, or from public or private research centers.
L'archive ouverte pluridisciplinaire HAL, est destinée au dépôt et à la diffusion de documents scientifiques de niveau recherche, publiés ou non, émanant des établissements d'enseignement et de recherche français ou étrangers, des laboratoires publics ou privés. 


\title{
Global Position-Feedback Tracking Control of Flexible-joint Robots
}

\author{
Sofia Avila-Becerril Antonio Loría Elena Panteley
}

\begin{abstract}
We solve the open problem of global tracking control of 2nd-degree under-actuated lossless (without friction) Lagrangian systems via position measurements only. For flexible-joint robots, we design a dynamic controller which is based on measurements of link and joint positions only. Then, approximate differentiation is used for link velocities and a simple Luenberger observer for rotor velocities. The main results constitute a significant extension of recent work on observerless output-feedback control of Lagrangian systems. Strictly speaking, we establish uniform global asymptotic stability for the closed loop system.
\end{abstract}

\section{INTRODUCTION}

The tracking control problem by output-feedback for Euler-Lagrange systems has been widely discussed in the literature -[18], [12], [4], [6], [15], [14]. For the system

$$
D(q) \ddot{q}+C(q, \dot{q}) \dot{q}+g(q)=u
$$

where $q \in \mathbb{R}^{n}$ and $\dot{q}$ denotes the generalized positions and velocities (respectively), the maps $D: \mathbb{R}^{n} \rightarrow \mathbb{R}^{n \times n}$ and $C: \mathbb{R}^{n} \times \mathbb{R}^{n} \rightarrow \mathbb{R}^{n \times n}$ corresponds to the inertia matrix and the Coriolis and centrifugal forces matrix, respectively, while $g: \mathbb{R}^{n} \rightarrow \mathbb{R}^{n}$ stands for the vector of forces derived from the potential energy function and $u \in \mathbb{R}^{n}$ is the vector of control inputs. Assuming that only generalized positions are available for measurement, the problem consist in design an internally stable control law that ensures that

$$
\lim _{t \rightarrow \infty} q(t) \rightarrow q_{d}(t), \quad \lim _{t \rightarrow \infty} \dot{q}(t) \rightarrow q_{d}(t) .
$$

One of the first results on this line was reported in [10] where, in the context of robot tracking, the authors consider state feedback controllers with a nonlinear observer that reproduces the whole dynamic ensuring local asymptotic stability. A large number of articles were published (including those cited above) in which different stability properties are established: semiglobal asymptotic stability -[9], [1], global asymptotic stability in part of the coordinates [18], global asymptotic stability -[12], [6], global exponential stability -[15], [14], etc. In [18], [12] however, it is assumed that the system has inherent dissipative forces (friction); for lossless systems, only [4], [6], [15], [14] establish uniform global asymptotic stability. In [4], however, the result applies to one-degree-of-freedom systems. It is only in [6], [15], [14]

\footnotetext{
*This work was realised while the first author was visiting LSS, under the sponsorship of the project ECOS Nord No. M14M02

S. Avila-Becerril is with Facultad de Ingeniería, UNAM, Mexico soavbececomunidad. unam.mx. A. Loria and E. Panteley are with the CNRS. LSS-CENTRALESUPELEC, 3 Rue Joliot Curie, 91192, France. E. Panteley is also with ITMO University, Kronverkskiy av. 49, Saint Petersburg, 197101, Russia. This article is supported by Government of Russian Federation (grant 074-U01).
}

that the long-standing open problem of establishing uniform global asymptotic stability for the closed-loop system, was fully solved, independently. See also [5].

For flexible-joint robots, the output-feedback tracking control is even more complex since the relative degree with respect to link positions is augmented by two relative to (1). In [11] the authors presented a result based on a semi-global nonlinear observer for the unmeasured variables, which needs only the link positions. In [19] the authors propose a robust output-feedback link position tracking control ensuring semi-global uniformly ultimately bounded link position tracking; and in the same spirit, in [3] based on a set of filters, removes the need of measuring link and actuator velocities, however the result relies on the restrictive assumption that the system is internally damped by viscous friction. In [17] a nonlinear observer-based certaintyequivalence tracking controller for Euler Lagrange systems is presented; global conditions are obtained under the assumption that one disposes of a controller that can be bounded by an affine function in the position times a polynomial of the velocities.

In [8] we presented a controller for flexible-joint robots which ensures uniform global asymptotic stability for the closed-loop system under the assumption that joint, but not link velocities, are measured. The controller in the latter is based on the more general results recently published in [7]. In the latter we established uniform global asymptotic stability for $n$ degrees of freedom systems with arbitrarilyhigh-relative-degree (with respect to generalized positions), without measurements of link velocities, the last includes under-actuated systems as the flexible joint robots. The control law is implemented through a chain of integrators in which the derivatives of virtual control inputs are replaced by approximate differentiation filters so that the result states a theoretical foundation for the use of the "dirty" derivatives.

In this paper, we follow the control method of [7], [8] and derive a certainty-equivalence controller which employs a reduced-order observer, in order to relax the assumption that joint velocities are measured. That is, under the assumption that only positions are available, we establish, for lossless flexible-joint robots, uniform global asymptotic stability in closed loop. As far as we know, there exists no other article in the literature establishing all this property under similar assumptions.

The rest of the paper is organized as follows: in Section II we formulate the global tracking problem and present some preliminary recent results. In Section III we present our main result. In Section IV we illustrate our findings in simulation, 
before concluding with some final remarks, in Section V.

\section{PROBLEM FORMULATION AND PRELIMINARIES}

According to [16], [2] joint flexibility in robot manipulators may be modelled using linear torsional springs; hence, if we denote by $K$ the joint stiffness ( $K=\infty$ implying no elasticity) the Lagrangian model is given by the equations

$$
\begin{aligned}
D\left(q_{1}\right) \ddot{q}_{1}+C\left(q_{1}, \dot{q}_{1}\right) \dot{q}_{1}+G\left(q_{1}\right) & =K\left(q_{2}-q_{1}\right) \\
J \ddot{q}_{2}+K\left(q_{2}-q_{1}\right) & =\tau
\end{aligned}
$$

where $q_{1} \in \mathbb{R}^{n}$ denote the under-actuated coordinates related to the links positions while $q_{2} \in \mathbb{R}^{n}$ represents the vector of motor shafts angles. The matrix $D\left(q_{1}\right)=D^{\top}\left(q_{1}\right)>$ 0 corresponds to the link-inertia matrix, $J$ stands for the diagonal rotor-inertia matrix, $C\left(q_{1}, \dot{q}_{1}\right) \dot{q}_{1}$ contains the terms due to the Coriolis and centrifugal forces, $G$ represents the gravitational forces vectors and $\tau$ corresponds to the physical control input. As it is customary, we make the following hypothesis on the model (2).

\section{Assumption 1:}

1) The inertia matrix $D\left(q_{1}\right)$ is positive definite and uniformly bounded; there exist positive real numbers $d_{m}$ and $d_{M}$ such that

$$
d_{m} \leq\left|D\left(q_{1}\right)\right| \leq d_{M}
$$

2) The matrix $C(x, y)$ is bounded in $x$ and linear in $y$. More precisely, there exists a saturation function sat: $\mathbb{R} \rightarrow \mathbb{R}$ such that, for all $x, y, z \in \mathbb{R}^{n}$,

$$
\begin{gathered}
|C(x, y)-C(z, y)| \leq k_{c} \operatorname{sat}(|x-z|)|y|, \quad|\operatorname{sat}(s)| \leq 1, \\
C(x, y) z=C(x, z) y,
\end{gathered}
$$

3) The matrix $\dot{D}\left(q_{1}\right)-2 C\left(q_{1}, \dot{q}_{1}\right)$ is skew symmetric for all $q_{1} \in \mathbb{R}^{n}$.

4) There exists $k_{v}$ such that function that represents the potential-energy forces, $G$, satisfies

$$
\exists k_{v}>0:\left|\frac{\partial G}{\partial q_{1}}\right| \leq k_{v}
$$

Under these conditions, we solve the global tracking control problem via position measurements only. More precisely, for any given reference trajectory $t \mapsto q_{d}$, twice continuously differentiable and bounded, i.e., such that $k_{\delta}>0$ such that

$$
\max \left\{\sup _{t \geq 0}\left|q_{d}(t)\right|, \sup _{t \geq 0}\left|\dot{q}_{d}(t)\right|, \sup _{t \geq 0}\left|\ddot{q}_{d}(t)\right|\right\} \leq k_{\delta}
$$

for some $k_{\delta}>0$, it is required to construct an outputfeedback dynamic controller

$$
\begin{aligned}
\dot{x}_{c} & =f\left(t, q_{1}, q_{2}, x_{c}\right) \\
u & =u\left(t, q_{1}, q_{2}, x_{c}\right)
\end{aligned}
$$

(that is, $x_{c} \in \mathbb{R}^{m}$ corresponds to the state of the controller) such that the origin of the closed-loop system is uniformly globally asymptotically stable. In order to solve this problem we follow the method of [7] which relies on the use of approximate differentiators to replace unavailable derivatives, such as velocity measurements.

For the sake of clarity, we first recall the main streamlines of the control method proposed in [7]. It concerns Lagrangian systems augmented by a chain of integrators; for the purposes of this paper we consider the case of two additional states, i.e.,

$$
\begin{aligned}
D\left(q_{1}\right) \ddot{q}_{1}+C\left(q_{1}, \dot{q}_{1}\right) \dot{q}_{1}+g\left(q_{1}\right) & =\xi_{1} \\
\dot{\xi}_{1} & =\xi_{2} \\
\dot{\xi}_{2} & =u .
\end{aligned}
$$

We remark that the model (2) may be transformed into (7) via a preliminary feedback and a change of variable. Indeed, this follows after a direct calculation, defining $g\left(q_{1}\right)=$ $G\left(q_{1}\right)+K q_{1}, \xi_{1}=K q_{2}, \xi_{2}=K \dot{q}_{2}$ and setting the input torque to

$$
\tau=\xi_{1}-K q_{1}+J K^{-1} u .
$$

Thus, the output-feedback tracking control problem boils down to stabilizing $\left(q_{1 d}, \dot{q}_{1 d}, 0,0\right)$ without measurement of $\dot{q}_{1}$ and $\xi_{2}$.

In [7] a controller that depends on all motor variables ( $q_{2}$ and $\dot{q}_{2}$ ) but not on link velocity measurements, $\dot{q}_{1}$ is proposed. In this paper, we relax the assumption that $\dot{q}_{2}$ is measurable. The output-feedback control design method proposed in [7] follows the rationale of backstepping control, in which unavailable derivatives are replaced by approximate differentiation. Hence, the starting point is to consider $\xi_{1}$ as a virtual control input in (7) and to design a virtual control law $\xi_{1}^{\star}$ which is set as reference for the integrator (7b) variable $\xi_{i}$, i.e.,

$\xi_{1}^{\star}=-k_{p_{0}} \tilde{q}_{1}-k_{d_{0}} \vartheta_{0}+D\left(q_{1}\right) \ddot{q}_{1 d}+C\left(q_{1}, \dot{q}_{1 d}\right) \dot{q}_{1 d}+g\left(q_{1}\right)$,

with $\tilde{q}_{1}:=q_{1}-q_{1 d}(t)$, whereas $\vartheta_{0}$ corresponds to the approximate differentiation of $\tilde{q}_{1}$ that is,

$$
\begin{array}{r}
\dot{q}_{c 0}=-a_{0} \vartheta_{0} \\
\vartheta_{0}=q_{c 0}+b_{0} \tilde{q}_{1} .
\end{array}
$$

Then, considering $\xi_{2}$ as a control input in (7b) we define

$$
\left.\begin{array}{c}
\xi_{2}^{\star}=-k_{p_{1}} \tilde{\xi}_{1}+k_{d_{1}} \vartheta_{1}+\dot{\xi}_{10}^{\star}, \\
\vartheta_{i}=q_{c i}+b_{i} \xi_{i}^{\star}+\zeta_{i} \\
\dot{q}_{c i}=-a_{i} \vartheta_{i} \\
\dot{\zeta}_{i}=-\left(k_{d_{i}}-\sigma_{i}\right) \tilde{\xi}_{i}-b_{i} \dot{\xi}_{i 0}^{\star}
\end{array}\right\} \forall i \in[1,2]
$$

where $\tilde{\xi}_{i}:=\xi_{i}-\xi_{i}^{\star}$, with $\sigma_{1}=0$ and $\sigma_{2}=b_{2} k_{p_{1}}$. It is proved in [7] that the origin $\{z=0\}$, with $z=$ $\left[\begin{array}{lllllll}\tilde{q}_{1}^{\top} & \dot{\tilde{q}}_{1}^{\top} & \vartheta_{0}^{\top} & \tilde{\xi}_{1}^{\top} & \tilde{\xi}_{2}^{\top} & \vartheta_{1}^{\top} & \vartheta_{2}^{\top}\end{array}\right]^{\top}$ and $\dot{\tilde{q}}_{1}:=\dot{q}_{1}-$ $\dot{q}_{1 d}(t)$, is uniformly globally asymptotically stable. Nevertheless, the controller (11) is based on the measurement of $\xi_{1}$ and $\xi_{2}$, which involves the measurement of motor shaft angular velocity, $\dot{q}_{2}$, since $\xi_{2}=K \dot{q}_{2}$. 
We remark that the dynamic controller (11) is designed on the basis of a backstepping design however, notice that the derivatives of $\xi_{1}^{\star}$ and $\xi_{2}^{\star}$ depend on unmeasurable variables such as velocities and accelerations hence, in place of $\dot{\xi}_{1}^{\star}$ and $\dot{\xi}_{2}^{\star}$, we employ approximate differentiation. This is implemented via the filters defined by (11a). To see this more clearly notice that these equations are equivalent to the Laplace representations:

$$
\vartheta_{i}=\frac{b_{i}}{s+a_{i}} \dot{\psi}_{i} \quad \Leftrightarrow \quad \vartheta_{i}=\frac{b_{i} s}{s+a_{i}} \psi_{i}
$$

with input $\dot{\psi}_{i}=\dot{\xi}_{i}^{\star}+\dot{\zeta}_{i} / b_{i}$ and $\psi_{0}=\tilde{q}$. This filter defines an output-strictly proper passive map $\vartheta_{i} \mapsto \dot{\psi}_{i}$ that is internally stable and input to state stable with respect to the input $\dot{\psi}_{i}$. However, it does not constitute an observer in the sense that one does not have $\vartheta_{i} \rightarrow \dot{\psi}_{i}$, in particular, $\vartheta_{0} \rightarrow \dot{\tilde{q}}$

\section{MAIN RESULT}

Our main statement (Proposition 1 below) is to establish a separation principle via position-feedback control of robot manipulators with flexible joints. We use a certaintyequivalence controller based on (11) and a simple reducedorder Luenberger observer. The latter is possible since the observer is designed only to estimate the unavailable state $\xi_{2}$, while other unmeasured variables such as link velocities, are "estimated" via dirty derivatives. While the design may be considered as naive, we remark that, as far as we know, there does not exist in the literature, a statement of uniform global asymptotic stability.

We define the reduced-order observer

$$
\begin{aligned}
& \dot{\hat{\xi}}_{1}=\hat{\xi}_{2}+\lambda_{1}\left(\xi_{1}-\hat{\xi}_{1}\right), \\
& \dot{\hat{\xi}}_{2}=u+\lambda_{2}\left(\xi_{1}-\hat{\xi}_{1}\right)
\end{aligned}
$$

where $\lambda_{1}$ and $\lambda_{2}$ are positive constant observer gains. Now, let the observer error $e=\left[\begin{array}{ll}e_{1}^{\top} & e_{2}^{\top}\end{array}\right]^{\top} \in \mathbb{R}^{2 n}$ be defined as $e=\xi-\hat{\xi}$ which, along (7) and (13), satisfies

$$
\begin{aligned}
& \dot{e}_{1}=e_{2}-\lambda_{1} e_{1} \\
& \dot{e}_{2}=-\lambda_{2} e_{1}
\end{aligned}
$$

Then, defining $\bar{\xi}=\hat{\xi}-\xi^{\star}$, consider the certainty equivalent controller

$$
\left.\begin{array}{c}
\xi_{2}^{\star}=-k_{p_{1}} \bar{\xi}_{1}+k_{d_{1}} \vartheta_{1}+\dot{\xi}_{10}^{\star}, \\
\vartheta_{i}=q_{c i}+b_{i} \xi_{i}^{\star}+\zeta_{i} \\
\dot{q}_{c i}=-a_{i} \vartheta_{i} \\
\dot{\zeta}_{i}=-\left(k_{d_{i}}-\sigma_{i}\right) \bar{\xi}_{i}-b_{i} \dot{\xi}_{i 0}^{\star}
\end{array}\right\} \forall i \in\{1,2\}
$$

where $k_{p i}$ and $k_{d i}$, which denote "proportional" and "derivative" control gains respectively, are positive constants. Then, we have the following.

Proposition 1: Let $q_{1 d}$ be given as in (4). Consider the system (2) under Assumption 1 in closed loop with the controller defined by Equations (8), (9) and (15). Then, the origin of the closed-loop system is uniformly globally asymptotically stable for sufficiently large control gains. Moreover, this holds for any positive observer gains $\lambda_{1}, \lambda_{2}$, independently of the control gains.

The proof is constructive, we derive explicit conditions on the control gains that imply uniform global asymptotic stability. It is organized in the following logical steps: first, we derive the closed-loop equations; then, we recognize that the closedloop dynamics has a cascaded form which stems from the application of certainty equivalence principle. The latter allows to invoke a cascades argument. Namely, that for a nonlinear time-varying system

$$
\begin{aligned}
& \dot{x}_{1}=f_{1}\left(t, x_{1}\right)+g(t, x) x_{2} \\
& \dot{x}_{2}=f_{2}\left(t, x_{2}\right)
\end{aligned}
$$

where $x_{1} \in \mathbb{R}^{n}, x_{2} \in \mathbb{R}^{m}, x:=\operatorname{col}\left[x_{1}, x_{2}\right]$ with $f_{1}, f_{2}$ and $g$ continuous and locally Lipschitz in $x$, uniformly in $t$, and $f_{1}$ continuously differentiable, it holds that if the respective origins of the subsystems (16b) and $\dot{x}_{1}=f_{1}\left(t, x_{1}\right)$ are uniformly globally asymptotically stable, it is sufficient and necessary for the origin of the overall system to possess the same property, that the solutions of (16) be uniformly globally bounded. This property is established along the proof-lines of [7, Theorem 5].

\section{A. The closed-loop equations}

The first error equation is obtained using the identity $\xi_{1}=\tilde{\xi}_{1}+\xi_{1}^{*}$, by replacing $\xi_{1}^{\star}$ from (9) in (7a) and adding $-C\left(q_{1}, \dot{q}_{1 d}\right) \dot{q}_{1}+C\left(q_{1}, \dot{q}_{1}\right) \dot{q}_{1 d}=0$ to the right-hand side of $(15 \mathrm{c})$. Then, we differentiate on both sides of (10b) and we use (10a) to obtain

$D\left(q_{1}\right) \ddot{\tilde{q}}_{1}+\left[C\left(q_{1}, \dot{q}_{1}\right)+C\left(q_{1}, \dot{q}_{1_{d}}\right)\right] \dot{\tilde{q}}_{1}+k_{p_{0}} \tilde{q}_{1}+k_{d_{0}} \vartheta_{0}=\tilde{\xi}_{1}$

$\dot{\vartheta}_{0}=-a_{0} \vartheta_{0}+b_{0} \dot{\tilde{q}}_{1}$.

On the other hand, note that the equations (7b)-(7c) are equivalent to

$$
\begin{aligned}
& \dot{\tilde{\xi}}_{1}=\xi_{2}^{\star}-\dot{\xi}_{1}^{\star}+\tilde{\xi}_{2} \\
& \dot{\tilde{\xi}}_{2}=u-\dot{\xi}_{2}^{\star}
\end{aligned}
$$

so, using the identity $\bar{\xi}_{i}=\tilde{\xi}_{i}-e_{i}$ for $i \in\{1,2\}$ as well as (15), we obtain

$$
\begin{aligned}
& \dot{\tilde{\xi}}_{1}=-k_{p 1} \tilde{\xi}_{1}+k_{d 1} \vartheta_{1}+\tilde{\xi}_{2}+k_{p 1} e_{1}-\left(\dot{\xi}_{1}^{\star}-\dot{\xi}_{10}^{\star}\right) \\
& \dot{\tilde{\xi}}_{2}=-k_{p 2} \tilde{\xi}_{2}+k_{d 2} \vartheta_{2}+k_{p 2} e_{2}-\left(\dot{\xi}_{2}^{\star}-\dot{\xi}_{20}^{\star}\right) \\
& \dot{\vartheta}_{i}=-a_{i} \vartheta_{i}-\left(k_{d i}-\sigma_{i}\right) \tilde{\xi}_{i}+\left(k_{d i}-\sigma_{i}\right) e_{i}+b_{i}\left(\dot{\xi}_{i}^{\star}-\dot{\xi}_{i 0}^{\star}\right)
\end{aligned}
$$

where $\sigma_{1}=0$.

Next, to compact the notation, we define $A:=$ $\operatorname{diag}\left\{a_{1}, a_{2}\right\} \otimes I_{n}, \quad B:=\operatorname{diag}\left\{b_{1}, b_{2}\right\} \otimes I_{n}, K_{d}:=$ $\operatorname{diag}\left\{k_{d_{1}}, k_{d_{2}}\right\} \otimes I_{n}, K_{p}:=\operatorname{diag}\left\{k_{p_{1}}, k_{p_{2}}\right\} \otimes I_{n}, K_{d}^{\prime}:=$ $\operatorname{diag}\left\{k_{d_{1}}, k_{d_{2}}-\sigma_{2}\right\} \otimes I_{n}$,

$$
K_{p}^{\prime}=\left[\begin{array}{cc}
k_{p_{1}} & -1 \\
0 & k_{p_{2}}
\end{array}\right], \quad L=\left[\begin{array}{cc}
\lambda_{1} & -1 \\
\lambda_{2} & 0
\end{array}\right]
$$


and defining $\vartheta^{\top}=\left[\begin{array}{ll}\vartheta_{1}^{\top} & \vartheta_{2}^{\top}\end{array}\right]$, then Equations (20) take the form

$$
\begin{aligned}
& \dot{\tilde{\xi}}=-K_{p}^{\prime} \tilde{\xi}+K_{d} \vartheta+K_{p} e-\left[\dot{\xi}^{\star}-\dot{\xi}_{0}^{\star}\right] \\
& \dot{\vartheta}=-A \vartheta-K_{d}^{\prime} \tilde{\xi}+K_{d}^{\prime} e+B\left[\dot{\xi}^{\star}-\dot{\xi}_{0}^{\star}\right] \\
& \dot{e}=-L e
\end{aligned}
$$

where the matrix $-L$ is Hurwitz.

These equations have a convenient structure. Firstly, the terms in brackets, $\left[\dot{\xi}^{\star}-\dot{\xi}_{0}^{\star}\right]$, vanish at the origin. This follows, on one hand, from the identity

$$
\dot{\xi}_{2}^{\star}-\dot{\xi}_{20}^{\star}=-k_{p_{1}} \bar{\xi}_{2}+\eta \bar{\xi}_{1}-\mu \vartheta_{1}+\beta\left[\dot{\xi}_{1}^{\star}-\dot{\xi}_{10}^{\star}\right]-\lambda_{1} e_{1}
$$

where $\mu=k_{d 1}\left(k_{p 1}+a_{1}\right), \eta=k_{p_{1}}^{2}-k_{d_{1}}^{2}$, and $\beta=k_{p_{1}}+$ $b_{1} k_{d 1}$ and $\bar{\xi}=\tilde{\xi}-e$. On the other hand, Assumption 1, together with (4), guarantees that $\dot{\xi}_{1}^{\star}\left(t, q, \dot{q}, \vartheta_{0}\right)$ is globally Lipschitz in the last two arguments, uniformly in $t$ and that is bounded in the first two arguments, so there exist nonnegative real numbers $\eta_{1}, \eta_{2}$ and $\eta_{3}$ as well as continuous saturation function sat $: \mathbb{R} \rightarrow \mathbb{R}$ such that $y \operatorname{sat}(y)>0$ for all $y \neq 0$ and $|\operatorname{sat}(y) \leq 1|$, and

$$
\left|\left[\dot{\xi}_{1}^{\star}-\dot{\xi}_{10}^{\star}\right]\right| \leq \eta_{1} \operatorname{sat}(|\tilde{q}|)+\eta_{2}\left|\dot{\tilde{q}}_{2}\right|+\eta_{3}\left|\vartheta_{0}\right| .
$$

Furthermore, the variable $\dot{\xi}_{2}^{\star}$ can be written as a function of $\tilde{\xi}, \vartheta, e$ and $\dot{\xi}_{1}^{\star}$; therefore, in view of (15a) and (20) we have

$$
\begin{aligned}
\dot{\xi}_{2}^{\star}= & \eta \tilde{\xi}_{1}-\mu \vartheta_{1}-\left(\eta+k_{p_{1}} \lambda_{1}\right) e_{1} \\
& -k_{p_{1}} \tilde{\xi}_{2}+k_{p_{1}} e_{2}+\beta\left[\dot{\xi}_{1}^{\star}-\dot{\xi}_{10}^{\star}\right]+\ddot{\xi}_{10}^{\star}
\end{aligned}
$$

So that equations (21) take the form

$$
\begin{aligned}
\dot{\tilde{\xi}}= & -\left(K_{p}^{\prime}+\Gamma_{1}\right) \tilde{\xi}+\left(K_{d}-\Gamma_{2}\right) \vartheta+\left(K_{p}+\Gamma_{4}\right) e \\
& -\Gamma_{3}\left[\dot{\xi}_{1}^{\star}-\dot{\xi}_{10}^{\star}\right] \\
\dot{\vartheta}= & -\left(K_{2}-B \Gamma_{1}\right) \tilde{\xi}-\left(A-B \Gamma_{2}\right) \vartheta+\left(K_{2}-B \Gamma_{4}\right) e \\
& +\left(B \Gamma_{3}\right)\left[\dot{\xi}_{1}^{\star}-\dot{\xi}_{10}^{\star}\right] \\
\dot{e}= & -L e
\end{aligned}
$$

where we defined

$$
\begin{aligned}
\Gamma_{1} & =\left[\begin{array}{cc}
0 & 0 \\
\eta & -k_{p_{1}}
\end{array}\right] \otimes I_{n}, \\
\Gamma_{2} & =\left[\begin{array}{cc}
0 & 0 \\
-\mu & 0
\end{array}\right] \otimes I_{n}, \\
\Gamma_{3} & =\left[\begin{array}{c}
1 \\
\beta
\end{array}\right] \otimes I_{n}, \\
\Gamma_{4} & =\left[\begin{array}{cc}
0 & 0 \\
k_{p_{1}} \lambda_{1}+\eta & -k_{p_{1}}
\end{array}\right] \otimes I_{n}, \\
K_{2} & =\left[\begin{array}{cc}
k_{d_{1}} & 0 \\
0 & k_{d_{2}}-\sigma_{2}
\end{array}\right] \otimes I_{n}
\end{aligned}
$$

Now, if we define $x_{1}:=\left[\begin{array}{cc}\tilde{\xi}^{\top} & \vartheta^{\top}\end{array}\right]^{\top}$ and $x_{2}:=e$ the system (25) becomes

$$
\begin{aligned}
& \dot{x}_{1}=\mathcal{A} x_{1}+\mathcal{B}\left[\dot{\xi}_{1}^{\star}-\dot{\xi}_{10}^{\star}\right]+K^{\prime} x_{2} \\
& \dot{x}_{2}=-L x_{2}
\end{aligned}
$$

where

$$
\begin{aligned}
\mathcal{A} & =\left[\begin{array}{cc}
-\left(K_{p}^{\prime}+\Gamma_{1}\right) & K_{d}-\Gamma_{2} \\
-\left(K_{2}-B \Gamma_{1}\right) & -\left(A-B \Gamma_{2}\right)
\end{array}\right], \mathcal{B}=\left[\begin{array}{c}
-\Gamma_{3} \\
B \Gamma_{3}
\end{array}\right] \\
K^{\prime} & =\left[\begin{array}{c}
K_{p}+\Gamma_{4} \\
K_{2}-B \Gamma_{1}
\end{array}\right] .
\end{aligned}
$$

This system has a convenient cascaded form, as (16). Moreover, since $L$ is Hurwitz by design, the origin of system (26b) is uniformly globally exponentially stable. Furthermore, after [7], the origin of

$$
\dot{x}_{1}=\mathcal{A} x_{1}+\mathcal{B}\left[\dot{\xi}_{1}^{\star}-\dot{\xi}_{10}^{\star}\right]
$$

is uniformly globally asymptotically stable for appropriate values of the control gains. In order to invoke the cascades argument, more precisely, [13, Lemma 2], it is left to prove that the solutions of (26a) are uniformly globally bounded.

\section{B. Boundedness of solutions}

Proposition 2: Let $L$ be Hurwitz, $k_{p_{1}}=k_{d_{1}}$ and assume that

$$
\begin{array}{r}
\min \left\{k_{p_{1}}, \frac{\left[k_{p_{2}}-k_{p_{1}}\right]}{\beta^{2}}, \frac{a_{1}}{b_{1}^{2}}, \frac{a_{2}}{b_{2}^{2} \beta^{2}}\right\}>\left[\eta_{2}^{2}+\eta_{3}^{2}\right] \\
{\left[k_{p_{2}}-k_{p_{1}}\right] \geq \max \left\{\frac{2}{k_{p_{1}}}, \frac{4 \mu^{2}}{a_{1}}\right\}, \quad a_{1} a_{2} \geq 2 b_{2}^{2} \mu^{2} .}
\end{array}
$$

Then, the solutions of the closed-loop system (17), (26) are uniformly globally bounded.

Proof. Let the Hurwitz property of $L$ generate positive definite matrices $P_{L}, Q_{L}$, satisfying $-Q_{L}:=\left(L^{\top} P_{L}+P_{L} L\right)$ and let $\kappa>0$ be a real constant. Then, consider the function $W: \mathbb{R}^{10 n} \rightarrow \mathbb{R}_{\geq 0}$, defined as

$$
W(x)=\kappa\left\|x_{1}\right\|^{2}+x_{2}^{\top} P_{L} x_{2} ;
$$

a direct computation shows that its total derivative along the trajectories of (26) yields

$$
\begin{aligned}
\dot{W}(x)= & \kappa\left[x_{1}^{\top}\left(\mathcal{A}+\mathcal{A}^{\top}\right) x_{1}+2 x_{1}^{\top} \mathcal{B}\left[\dot{\xi}_{1}^{\star}-\dot{\xi}_{10}^{\star}\right]+\right. \\
& \left.+2 x^{\top} P K^{\prime} x_{2}\right]-x_{2}^{\top} Q_{L} x_{2} .
\end{aligned}
$$

Now, let $Q=-\left(\mathcal{A}^{\top}+\mathcal{A}\right)$; we see that, since $k_{p_{1}}=k_{d_{1}}$,

$$
Q=\left[\begin{array}{cccc}
2 k_{p_{1}} & -1 & 0 & 0 \\
-1 & 2\left[k_{p_{2}}-k_{p_{1}}\right] & -\mu & 0 \\
0 & -\mu & 2 a_{1} & -b_{2} \mu \\
0 & 0 & -b_{2} \mu & 2 a_{2}
\end{array}\right] \otimes I_{n} .
$$

Hence, defining $c:=\left|K^{\prime}\right|, \lambda:=|L|$, using the CauchySchwartz and the triangle inequalities, we obtain

$$
\begin{aligned}
\dot{W} \leq & -\kappa x_{1}^{\top} Q x_{1}+2 \kappa x_{1}^{\top} \mathcal{B}\left[\dot{\xi}_{1}^{\star}-\dot{\xi}_{10}^{\star}\right]+2 \kappa c\left|x_{1}\right|\left|x_{2}\right|-\lambda\left|x_{2}\right|^{2} \\
\leq & -\kappa x_{1}^{\top} Q x_{1}+2 \kappa x_{1}^{\top} \mathcal{B}\left[\dot{\xi}_{1}^{\star}-\dot{\xi}_{10}^{\star}\right]+\frac{\kappa c}{\epsilon}\left|x_{1}\right|^{2} \\
& -(\lambda-\kappa c \epsilon)\left|x_{2}\right|^{2}
\end{aligned}
$$

Now, notice that the matrix $Q-1 / 2 \operatorname{diag}\{Q\}$ is positive semidefinite if so are

$$
\left[\begin{array}{cc}
k_{p_{1}} & -1 \\
-1 & 1 / 2\left[k_{p_{2}}-k_{p_{1}}\right]
\end{array}\right]
$$




$$
\left[\begin{array}{cc}
1 / 2\left[k_{p_{2}}-k_{p_{1}}\right] & -\mu \\
-\mu & 1 / 2 a_{1}
\end{array}\right],\left[\begin{array}{cc}
1 / 2 a_{1} & -b_{2} \mu \\
-b_{2} \mu & a_{2}
\end{array}\right],
$$

which hold in view of (28). Therefore, under these conditions, we see that

$$
\begin{aligned}
\dot{W} \leq & -\kappa x_{1}^{\top}\left(\frac{1}{2} \operatorname{diag}\{Q\}-\frac{c}{\epsilon} I\right) x_{1}+2 \kappa x_{1}^{\top} \mathcal{B}\left[\dot{\xi}_{1}^{\star}-\dot{\xi}_{10}^{\star}\right] \\
& -(\lambda-\kappa c \epsilon)\left|x_{2}\right|^{2}
\end{aligned}
$$

Notice that the factors of $\left|x_{1}\right|^{2}$ and $\left|x_{2}\right|^{2}$ are negative for any $\lambda>0$, sufficiently large values of $\epsilon$ and $\kappa:=1 / \epsilon^{2}$. On the other hand, under Assumption 1, from Inequality (23) and the triangle inequality, it follows that

$$
\begin{aligned}
& x_{1}^{\top} \mathcal{B}\left[\dot{\xi}_{1}^{\star}-\dot{\xi}_{10}^{\star}\right] \leq \eta_{1}\left(\left|\tilde{\xi}_{1}\right|+\beta_{1}\left|\tilde{\xi}_{2}\right|+b_{1}\left|\vartheta_{1}\right|+b_{2} \beta_{1}\left|\vartheta_{2}\right|\right) \\
& \quad+\frac{1}{2}\left(\eta_{2}^{2}+\eta_{3}^{2}\right)\left(\left|\tilde{\xi}_{1}\right|^{2}+\beta_{1}^{2}\left|\tilde{\xi}_{2}\right|^{2}+b_{1}^{2}\left|\vartheta_{1}^{2}\right|+b_{2}^{2} \beta_{1}^{2}\left|\vartheta_{2}\right|^{2}\right) \\
& \quad+2\left|\dot{\tilde{q}}_{1}\right|^{2}+2\left|\vartheta_{0}\right|^{2} .
\end{aligned}
$$

Next, let $\mathcal{V}: \mathbb{R}_{\geq 0} \times \mathbb{R}^{13 n} \rightarrow \mathbb{R}_{\geq 0}$ be defined as $V\left(t, \tilde{q}_{1}, \dot{\tilde{q}}_{1}, \vartheta_{0}\right)+W(x)$, where

$$
V=\frac{\kappa}{2}\left(\dot{\tilde{q}}_{1}^{\top} D\left(\tilde{q}_{1}+q_{1 d}(t)\right) \dot{\tilde{q}}_{1}+k_{p_{0}}\left|\tilde{q}_{1}\right|^{2}+\frac{k_{d_{0}}}{b_{0}}\left|\vartheta_{0}\right|^{2}\right)
$$

The total derivative of $\mathcal{V}:=V+W$ along the trajectories of (17) and (26) yields

$\dot{\mathcal{V}} \leq-\frac{a_{0} k_{d_{0}} \kappa}{b_{0}}\left|\vartheta_{0}\right|^{2}+k_{c} k_{\delta} \kappa\left|\dot{\tilde{q}}_{1}\right|^{2}+\kappa \dot{\tilde{q}}_{1}^{\top} \tilde{\xi}_{1}-(\lambda-\kappa c \epsilon)\left|x_{2}\right|^{2}$

$-\kappa \dot{\tilde{q}}_{1} e_{1}-\kappa x_{1}^{\top}\left(\frac{1}{2} \operatorname{diag}\{Q\}-{ }_{-}^{c} I\right) x_{1}+2 \kappa x_{1}^{\top} \mathcal{B}\left[\dot{\xi}_{1}^{\star}-\dot{\xi}_{10}^{\star}\right]$

that in view of (32) can be expressed as

$$
\begin{aligned}
& \dot{\mathcal{V}} \leq-\kappa x_{1}^{\top}\left(\frac{1}{2} \operatorname{diag}\{Q\}-\frac{c}{\epsilon} I\right) x_{1}-\left(\lambda-\frac{\kappa}{2}-\kappa c \epsilon\right)\left|x_{2}\right|^{2} \\
& +\frac{\kappa}{2}\left|\tilde{\xi}_{1}\right|^{2}+2 \kappa \eta_{1}\left(\left|\tilde{\xi}_{1}\right|+\beta_{1}\left|\tilde{\xi}_{2}\right|+b_{1}\left|\vartheta_{1}\right|+b_{2} \beta_{1}\left|\vartheta_{2}\right|\right) \\
& +\kappa\left(\eta_{2}^{2}+\eta_{3}^{2}\right)\left(\left|\tilde{\xi}_{1}\right|^{2}+\beta_{1}^{2}\left|\tilde{\xi}_{2}\right|^{2}+b_{1}^{2}\left|\vartheta_{1}^{2}\right|+b_{2}^{2} \beta_{1}^{2}\left|\vartheta_{2}\right|^{2}\right) \\
& +\kappa\left(\frac{7}{2}+k_{c} k_{\delta}\right)\left|\dot{\tilde{q}}_{1}\right|^{2}-\kappa\left(\frac{a_{0} k_{d_{0}}}{b_{0}}-2\right)\left|\vartheta_{0}\right|^{2}
\end{aligned}
$$

By assumption, the quadratic term in $x_{1}$ is negative definite and dominates over all positive terms, except on that involving $\left|\dot{\tilde{q}}_{1}\right|^{2}$.

The rest of the proof of boundedness follows as for Theorems 5 and 6 of [7]. Roughly speaking in the latter it is established that, even though $\vartheta_{0} \nrightarrow \rightarrow \dot{\tilde{q}}_{1}$, the outputinput gain of the filter (12) is finite. More precisely, to any monotonically increasing sequence $\left\{\left[\left|\dot{\tilde{q}}_{1}\right|^{2}\right]_{i}\right\}$ where $\left[\left|\dot{\tilde{q}}_{1}\right|^{2}\right]_{i}=\left|\dot{\tilde{q}}_{1}\left(t_{i}\right)\right|^{2}$, corresponds a sequence $\left\{\left[\left|\vartheta_{0}\right|^{2}\right]_{i}\right\}$ which increases at the same rate. Therefore, if $\left\{\left|\dot{\tilde{q}}_{1}(t)\right|^{2}\right\}$ grows unboundedly so does $\left\{\left|\vartheta_{0}(t)\right|^{2}\right\}$ hence, sequentially, $\dot{\mathcal{V}}\left(t_{i}\right)$ becomes non-positive and $\left\{\mathcal{V}\left(t_{i}\right)\right\}$ is bounded. Rigorous proof along these arguments is provided in [7].

Finally, the statement of Proposition 1 follows invoking [13, Lemma 2].

\section{Simulations Results}

In order to evaluate the global characteristic of the controller (15) we present some numerical simulations. To this end, we consider a two-degrees-of-freedom planar flexible joint robot whose model is given by

$$
\begin{aligned}
D\left(q_{1}\right) & =\left[\begin{array}{cc}
8.77+1.02 c_{12} & 0.76+0.51 c_{12} \\
0.76+0.51 c_{12} & 0.62
\end{array}\right] \\
C\left(q_{1}, \dot{q}_{1}\right) & =\left[\begin{array}{cc}
-0.51 s_{12} \dot{q}_{12} & -0.51 s_{12}\left(\dot{q}_{11}+\dot{q}_{12}\right) \\
0.51 s_{12} \dot{q}_{11} & 0
\end{array}\right]
\end{aligned}
$$

where $c_{12}$ and $s_{12}$ denotes the $\cos \left(q_{12}\right)$ and $\sin \left(q_{12}\right)$ respectively. Furthermore, the joint stiffness matrix is $K=\operatorname{diag}\{10000,10000\}$ while the rotor inertia matrix is $J=\operatorname{diag}\{0.1,0.1\}$. The gravitational forces vector $g\left(q_{1}\right)^{\top}=$ $\left[9.81\left(7.6 s_{11}+0.63 s_{11,12} \quad 9.81\left(0.63 s_{11,12}\right)\right]\right.$ with $s_{11,12}=$ $\sin \left(q_{11}+q_{12}\right)$. And we have set the desired value of the under-actuated coordinates to $q_{1 d}(t):=\sin (\omega t) \mathrm{rad}$, with $\omega_{1}=2 \mathrm{rad} / \mathrm{s}$.

With the aim at illustrating the global character of the stability enhanced by our controller, we purposefully performed a simulation test with an unrealistic $5000 \%$ of initial error. The control parameters for the filters were set to $a_{0}=500$, $b_{0}=500 a_{1}=2500, b_{1}=5, a_{2}=5000, b_{2}=5$, the control gains were fixed in $k_{p 0}=400, k_{p 1}=1, k_{p 2}=8000$ and $k_{d 0}=35, k_{d 1}=500, k_{d 2}=10$, while the observer parameters $\lambda_{1}=10$ and $\lambda_{2}=20$. In Figures 1 and 2 we show the position and the velocity of the under-actuated coordinates $q_{1}$ and $\dot{q}_{1}$ respectively. These graphs show the proper functioning of the controller and its global property. In Figure 3 we depict the observer performance.

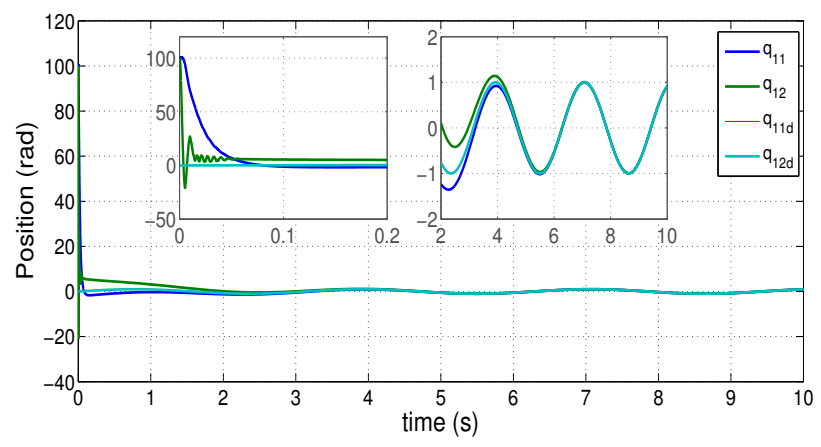

Fig. 1. Link angular positions and reference

\section{CONCLUSIONS}

In this paper we make an extension of the results in [7] in a way that the output-feedback problem can be solved based only in the measurement of the link position preserving the uniformly globally asymptotic stability property for the closed-loop system. The result is based on the use of a Luenberger observer and in particular, solves the problem for the flexible joint robots under the conditions: the system is lossless and motor velocities are unmeasured. 


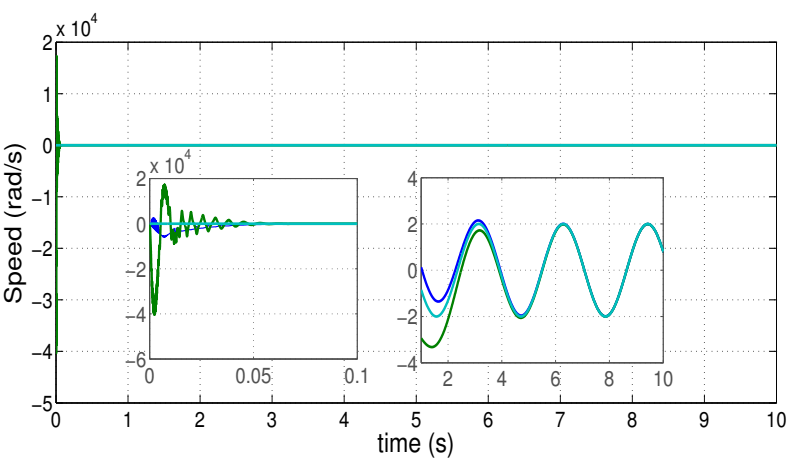

Fig. 2. Link angular velocities and reference

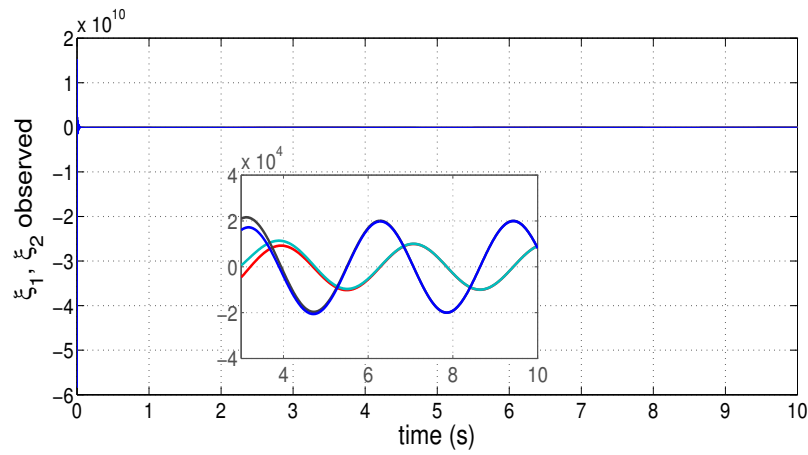

Fig. 3. Observed variables $\hat{\xi}_{1}, \hat{\xi}_{2}$ and references.

\section{REFERENCES}

[1] I. V. Burkov. Mechanical system stabilization via differential observer. In IFAC Conference on System Structure and Control, pages 532-535, Nantes, France, 1995.

[2] I. V. Burkov and A. T. Zaremba. Dynamics of elastic manipulators with electric drives. Izv. Akad. Nauk SSSR Mekh. Tverd. Tela, 22(1):57-64, 1987. Engl. transl. in Mechanics of Solids, Allerton Press.

[3] S. Y. Lim, D. M Dawson, J. Hu, and M. S. De Queiroz. An adaptive link position tracking controller for rigid-link flexible-joint robots without velocity measurements. Systems, Man, and Cybernetics, Part B: Cybernetics, IEEE Transactions on, 27(3):412-427, 1997.

[4] A. Loría. Global tracking control of one degree of freedom EulerLagrange systems without velocity measurements. European J. of Contr., 2(2), 1996.
[5] A. Loría. Observer-less output feedback global tracking control of lossless lagrangian systems. e-print no. arXiv:1307.4659, June 2013. Available from http://arxiv.org/abs/1307.4659 .

[6] A. Loría. Uniform global position feedback tracking control of mechanical systems. In Proc. IEEE American Control Conference, pages 5722-5727, Washington, D.C., 2013. DOI: 10.1109/ACC.2013.6580734.

[7] A. Loría. Observers are unnecessary for output-feedback control of Lagrangian systems. IEEE Trans. Automat. Control, 2015. Prepublished online. DOI: 10.1109/TAC.2015.2446831.

[8] A. Loría and S. Avila-Becerril. Output-feedback global tracking control of robot manipulators with flexible joints. In Proc. IEEE American Control Conference, pages 4032-4037, Portland, Oregon, 2014. DOI: 10.1109/ACC.2014.6858900.

[9] A. Loría and R. Ortega. On tracking control of rigid and flexible joints robots. Appl. Math. and Comp. Sci., special issue on Mathematical Methods in Robotics, K. Tchon and A. Gosiewsky, eds, 5(2):101-113, 1995.

[10] S. Nicosia and P. Tomei. Robot control by using only joint position measurement. IEEE Trans. on Automat. Contr., 35-9:1058-1061, 1990.

[11] S. Nicosia and P. Tomei. A tracking controller for flexible joint robots using only link position feedback. IEEE Trans. on Automat. Contr. 40(5):885-890, 1995.

[12] E. V. L. Nunes and L. Hsu. Global tracking for robot manipulators using a simple causal PD controller plus feedforward. Robotica, 28(1):23-34, 2010. DOI:10.1017/S0263574709005529.

[13] E. Panteley and A. Loría. Growth rate conditions for stability of cascaded time-varying systems. Automatica, 37(3):453-460, 2001.

[14] J.G. Romero, R. Ortega, and I. Sarras. A globally exponentially stable tracking controller for mechanical systems using position feedback. Automatic Control, IEEE Transactions on, 60(3):818-823, March 2015.

[15] J. G. Romero-Velázquez, I. Sarras, and R. Ortega. A globally exponentially stable tracking controller for mechanical systems using position feedback. In Proc. IEEE American Control Conference, pages 4976-4981, 2013

[16] M. Spong. Modeling and control of elastic joint robots. ASME J. Dyn. Syst. Meas. Contr., 109:310-319, 1987.

[17] Ø. N. Stamnes, O. M. Aamo, and G-O. Kaasa. Global output feedback tracking control of Euler-Lagrange systems. In Proc of 18th IFAC Word Congress, pages 215-220, 2011.

[18] E. Zergeroglu, D. M. Dawson, M. S. de Queiroz, and M. Krstić. On global output feedback tracking control of robot manipulators. In Proc. 39th. IEEE Conf. Decision Contr., pages 5073-5078, Sydney, Australia, 2000.

[19] Y. Zhu, D. Dawson, T. Burg, and J. Hu. A cheap output feedback tracking controller with robustness: the rlfj problem. In Robotics and Automation, 1996. Proceedings., 1996 IEEE International Conference on, volume 1, pages 939-944 vol.1, 1996. 